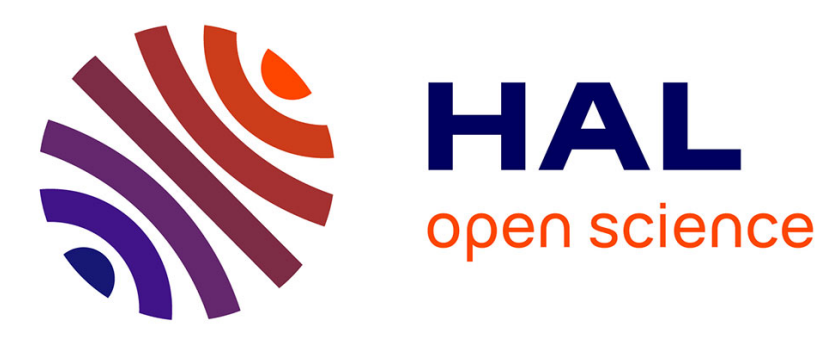

\title{
Utilisations de l'effet Josephson alternatif T. Pech
}

\section{- To cite this version:}

T. Pech. Utilisations de l'effet Josephson alternatif. Revue de Physique Appliquée, 1971, 6 (4), pp.535-542. 10.1051/rphysap:0197100604053500 . jpa-00243584

\section{HAL Id: jpa-00243584 https://hal.science/jpa-00243584}

Submitted on 1 Jan 1971

HAL is a multi-disciplinary open access archive for the deposit and dissemination of scientific research documents, whether they are published or not. The documents may come from teaching and research institutions in France or abroad, or from public or private research centers.
L'archive ouverte pluridisciplinaire HAL, est destinée au dépôt et à la diffusion de documents scientifiques de niveau recherche, publiés ou non, émanant des établissements d'enseignement et de recherche français ou étrangers, des laboratoires publics ou privés. 


\title{
UTILISATIONS DE L'EFFET JOSEPHSON ALTERNATIF
}

\author{
T. PECH
}

L. C. I. E., 33, Avenue du Général-Leclerc, 92-Fontenay-aux-Roses

\begin{abstract}
Résumé. - L'auteur présente les mécanismes physiques qui expliquent la génération de rayonnements électromagnétiques par des jonctions Josephson, ainsi que le comportement de celles-ci en présence d'un ou de plusieurs rayonnements appliqués de l'extérieur. Les caractéristiques principales des phénomènes expérimentaux sont discutées pour chaque cas; elles conduisent à divers types d'utilisations tels que source et détecteur de rayonnement, dispositif pour la surveillance des piles étalon de tension, oscillateur, mélangeur. Pour terminer, la description d'un thermomètre de bruit illustre une utilisation toute particulière des jonctions Josephson.
\end{abstract}

Abstract. - The physical phenomena taking place in Josephson junctions and responsible for the generation of electromagnetic radiation as well as for their behaviour in the presence of one or two external radiations are discussed. The principal characteristics of the experimental phenomena are discussed for each case. These characteristics lead to various applications of the Josephson junctions, such as generator and detector of radiation, device to maintain standards of electromotive force, oscillator, mixer. A rather particular type of application is illustrated by the description of a noise thermometer.

Toutes les applications qui seront discutées dans cette partie sont essentiellement basées sur la relation de_Josephson

$$
v=\frac{2 \mathrm{eV}}{h}
$$

laquelle exprime qu'une jonction polarisée à la tension $V$ est traversée par un supracourant alternatif de fréquence $v$ ( $e$ est la valeur absolue de la charge de l'électron et $h$ la constante de Planck).

Les équations bien connues qui décrivent complètement les propriétés électromagnétiques des jonctions Josephson, et dont nous aurons besoin dans la suite, sont les suivantes :

$$
\begin{aligned}
j(r, t) & =j_{1} \sin \varphi(r, t) \\
\frac{\partial \varphi(r, t)}{\partial t} & =\frac{2 e V(r, t)}{\hbar} \\
\nabla \varphi(r, t) & =\frac{2 e d}{\hbar c}(H \times \mathbf{n}) .
\end{aligned}
$$

L'équation (1) exprime la densité de courant à travers la barrière isolante en fonction de la position $r$ et du temps $t, \varphi$ étant la différence de phase des fonctions d'onde relatives aux deux supraconducteurs qui constituent la jonction. La constante $j_{1}$ dépend de la température et de l'épaisseur de la barrière. (2) indique la variation temporelle de $\varphi$, où $V(r, t)$ est la tension totale aux bornes de la jonction au point considéré, et $\hbar=h / 2 \pi$. La variation spatiale de la phase dépend d'une intégrale curviligne du potentiel vecteur A. Pour une jonction tunnel l'intégration peut être effectuée en termes du champ magnétique $H$ dans le plan de la barrière, de la profondeur de pénétration $\lambda$ du supraconducteur et de l'épaisseur $l$ de la barrière isolante. Les calculs conduisent à l'équation (3), exprimée en unités de Gauss, où $d=2 \lambda+l, c$ est la vitesse de la lumière et $\mathbf{n}$ est le vecteur unitaire perpendiculaire au plan de la barrière.

1. Génération de rayonnements hyperfréquences. Dans le cas d'une tension de polarisation continue $V_{0}$ et d'un champ magnétique $H_{0}$ appliqué de l'extérieur suivant la direction $y$ (Fig. 1) l'équation (1) devient

$$
j=j_{1} \sin \left(\omega_{0} t-k z+\varphi_{0}\right)
$$

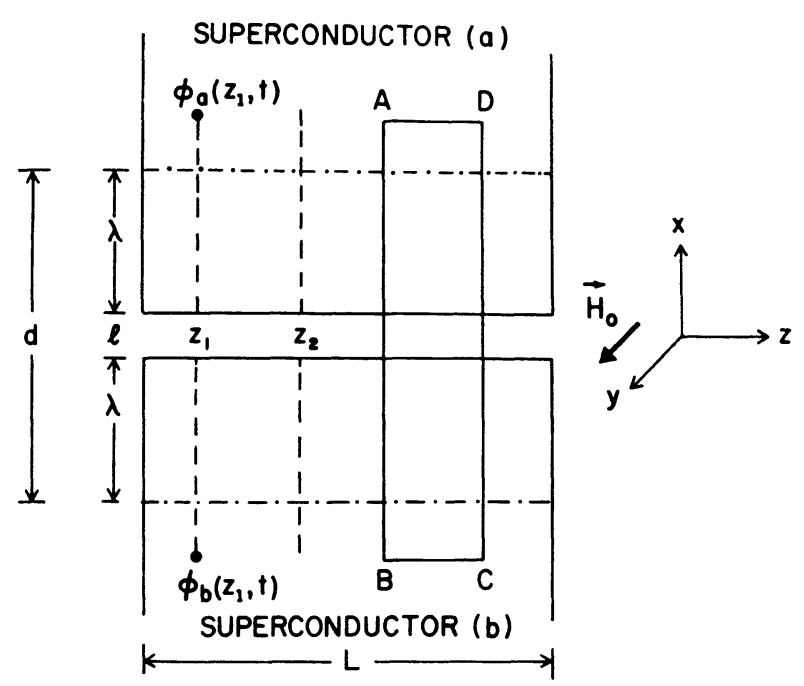

Fig. 1. - Coupe d'une jonction montrant la différence de phase $\varphi\left(z_{1}, t\right)=\Phi_{\mathrm{a}}\left(z_{1}, t\right)-\Phi_{\mathrm{b}}\left(z_{1}, t\right)$, la profondeur de pénétration $\lambda$ et l'orientation du champ magnétique extérieur $H_{0}$. La jonction est dans le plan $y-z ; l$ est l'épaisseur de la barrière isolante et $L$ est la longueur de la jonction. 
où

$$
k=\frac{2 e d H_{0}}{\hbar c}
$$

et

$$
\omega_{0}=\frac{2 e V_{0}}{h}=2 \pi v_{0} .
$$

(4) décrit l'onde de densité de courant évoluant à travers la barrière suivant la direction $z$, perpendiculaire à $H_{0}$.

Il est utile de rappeler brièvement les premières études de mise en évidence directe du courant Josephson alternatif (sans irradiation hyperfréquence appliquée de l'extérieur), effectuées par Eck, Scalapino et Taylor [1] et puis par Langenberg et ses collaborateurs [2].

Il s'agit essentiellement de l'étude de la structure des caractéristiques $I-V$ de la jonction, due à l'interaction du courant Josephson alternatif et des champs électromagnétiques produits dans la barrière par le courant Josephson alternatif.

Dans les conditions relatives à (4), la vitesse de phase de l'onde de densité de courant est

$$
\frac{\omega_{0}}{k}=\frac{V_{0}}{H_{0} d} c .
$$

Les ondes magnétiques transversales qui se propagent dans la barrière isolante peuvent être facilement calculées avec l'hypothèse simplificatrice de deux supraconducteurs semi-infinis séparés par une barrière très mince $(10$ à $20 \AA)$. La vitesse de phase $c^{\prime}$ de ces ondes s'exprime alors en fonction des paramètres de la jonction

$$
c^{\prime}=c \sqrt{\frac{l}{\varepsilon d}}
$$

où $\varepsilon$ est la constante diélectrique relative de la région isolante.

Dans le cas d'un système faiblement couplé, le calcul de la tension alternative $v$ qui prend naissance aux bornes de la jonction conduit, avec l'hypothèse de $v_{0} / V_{0} \ll 1$ et en représentant les pertes par $Q$, à

$$
v=v_{0} \cos \left(\omega_{0} t-k z+\theta\right)
$$

où

$$
v_{0}=\frac{j_{1}\left(4 \pi l / \varepsilon \omega_{0}\right)}{\left\{\left[1-\left(k c^{\prime} / \omega_{0}\right)^{2}\right]^{2}+(1 / Q)^{2}\right\}^{1 / 2}}
$$

et

$$
\theta=\tan ^{-1}\left[\frac{1 / Q}{1-\left(k c^{\prime} / \omega_{0}\right)^{2}}\right]+\varphi_{0}
$$

Maintenant, si l'on tient compte du fait que, suivant (2), la variation temporelle de $\varphi$ dépend de la tension totale, $V=V_{0}+v$ aux bornes de la jonction, l'expression de $j$ qu'on peut obtenir à partir de (1) devient :

$j=j_{1} \sin \left[\omega_{0} t-k z+\frac{v_{0}}{V_{0}} \sin \left(\omega_{0} t-k z+\theta\right)+\varphi_{0}\right]$.

La partie continue de (7) est alors approximativement

$$
\begin{aligned}
j_{\text {cont }}=j_{1}\left(\frac{2 \pi l j_{1}}{\varepsilon \omega_{0} V_{0}}\right) & \times \\
& \times \frac{1 / Q}{\left[1-\left(k c^{\prime} / \omega_{0}\right)^{2}\right]^{2}+1(/ Q)^{2}} .
\end{aligned}
$$

L'expression (8) montre que le courant continu résultant a une allure de résonance. Le maximum se produit quand la vitesse de phase (5) de l'onde de densité de courant devient égale à la vitesse de phase (6) des ondes magnétiques transversales. De la condition $\omega_{0} / k=c^{\prime}$ on déduit alors la tension de polarisation continue, $V_{0}=V_{\mathrm{p}}$, pour laquelle il se produit un maximum pour le courant continu :

$$
V_{\mathrm{p}}=\left(\frac{l d}{\varepsilon}\right)^{1 / 2} H_{0} .
$$

Si l'on tient compte des effets produits par le fait que la longueur de la jonction n'est pas infinie, l'expression de la densité du courant continu moyenne dans l'espace est plus compliquée que (8), en particulier à cause des modes de tension qui varient comme $\cos n \pi z / L$, où $L$ est la longueur de la jonction (dans la direction $z$ ) et $n$ est un nombre entier.

Différentes situations peuvent se produire suivant : a) les dimensions géométriques de la jonction étudiée, b) le degré d'uniformité de sa couche de barrière et de la distribution du champ magnétique à l'intérieur, et c) les valeurs de $H_{0}$ et $V_{0}$ utilisées. Pour les diverses situations, l'accord entre les calculs et les résultats expérimentaux semble être très satisfaisant. S'il s'agit d'une situation où l'effet de la non-uniformité est prépondérant, on peut obtenir des courbes $I-V$ présentant une résonance, comme sur la figure 2, extraite de [2], pour laquelle à la résonance

$$
j_{\text {cont }}=\frac{4 \pi l j_{1}^{2}}{\varepsilon \omega_{0} V_{0}}\left(\frac{\omega_{0} L}{4 c^{\prime}}\right) .
$$

En revanche, une succession de marches d'escalier a été observée sur la caractéristique $I-V$ pour la première fois par Fiske. En effet, les jonctions ayant des caractéristiques uniformes, et soumises à un champ $H_{0}$ faible peuvent conduire à une courbe $I-V$ de cette nature, comme sur la figure 3, extraite de [2] également. Dans ce cas, les marches se manifestent à des tensions régulièrement espacées

$$
n V^{\prime}=n \frac{h c^{\prime}}{4 e L} .
$$




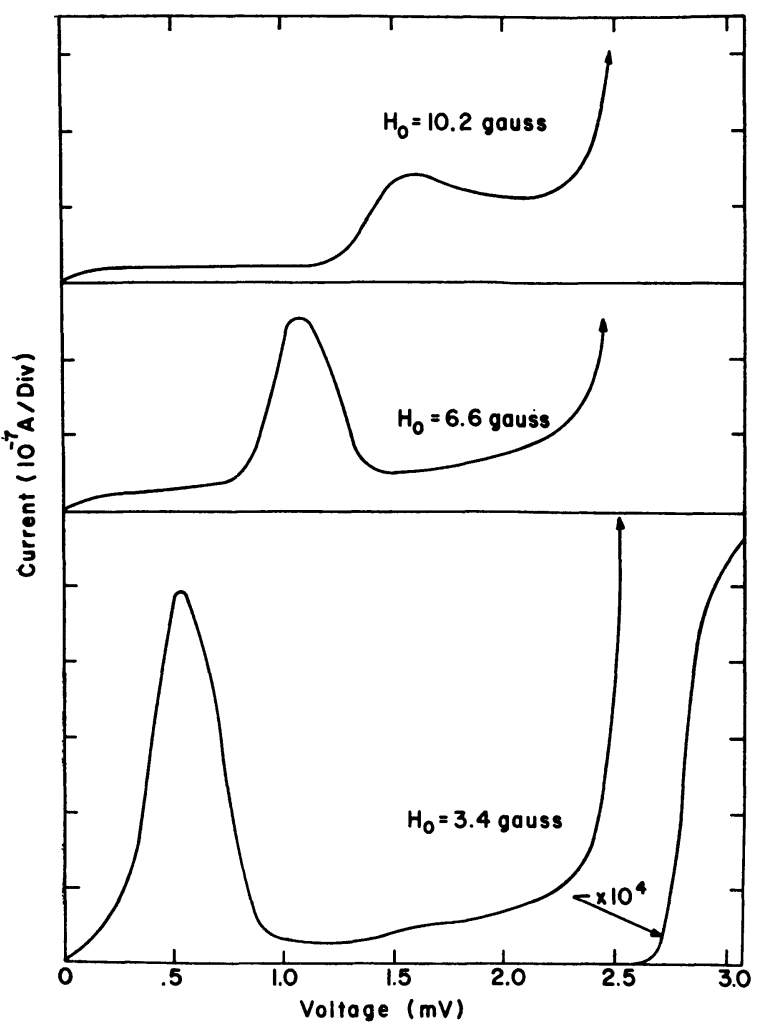

Fig. 2. - Caractéristiques $I-V$ d'une jonction $\mathrm{Pb}-\mathrm{PbO}-\mathrm{Pb}$ à $1,2{ }^{\circ} \mathrm{K}$ présentant une allure de résonance variable en fonction du champ magnétique appliqué.

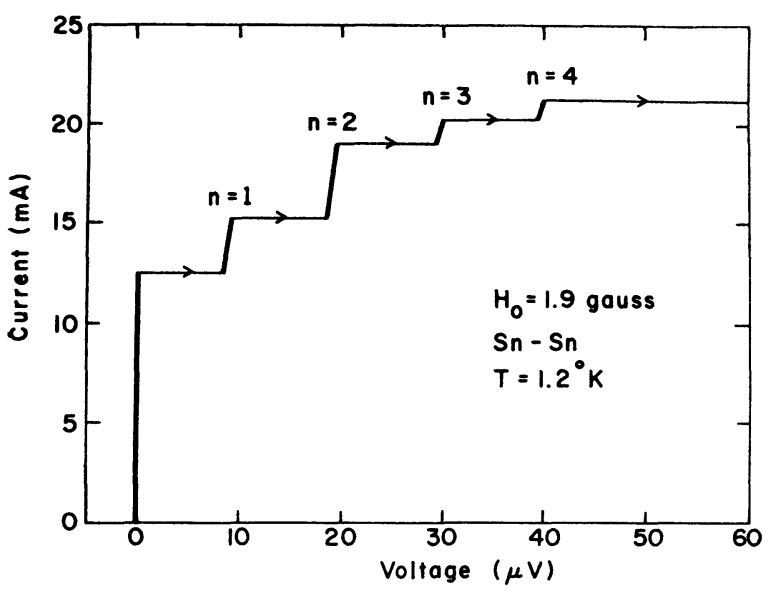

Fig. 3. - Caractéristique $I-V$ d'une jonction Sn-SnO-Sn présentant des marches d'escalier régulièrement espacées.

Nous constatons donc qu'en présence d'un champ magnétique continu, appliqué de l'extérieur, des phénomènes de résonance peuvent prendre naissance dans des jonctions, ces phénomènes étant très fortement influencés par les divers paramètres des jonctions. En conséquence, on peut espérer qu'un rayonnement électromagnétique, de fréquence $v=2 \mathrm{eV} / \mathrm{h}$, est détectable chaque fois que l'interaction du courant Josephson alternatif avec les ondes magnétiques transversales produit une structure caractéristique sur les courbes $I-V$ continus de la jonction.
Quelle est la puissance de rayonnement qu'on peut espérer obtenir ?

La valeur moyenne $E_{n}$ de l'énergie électromagnétique stockée dans le $n$-ième mode de résonance de la jonction peut être estimée à l'aide de $: a$ ) la puissance d'entrée continue de la jonction $P_{\text {cont }}=I . V$, déterminée par le point de fonctionnement $I-V$ continus ; avec une valeur de $I=15 \mathrm{~mA}$, caractéristique pour une jonction tunnel, et à $V=20 \mu \mathrm{V}$ (correspondant à $v=10 \mathrm{GHz}) P_{\text {cont }}$ n'est guère que $\sim 3 \times 10^{-7} \mathrm{~W}$; b) de la valeur de $Q_{n}$ appartenant au mode $n$ qu'on peut évaluer à partir de la pente de la caractéristique $I-V$ au voisinage de la $n$-ième marche.

Si l'on tient compte du fait qu'en pratique plusieurs modes, $m$, peuvent être excités simultanément, on obtient approximativement

$$
E_{n} \simeq \frac{P_{\text {cont }} Q_{n}}{m \cdot \omega_{n}}
$$

Pour une jonction de longueur $L$, la puissance hyperfréquence qui apparaît aux bornes de la jonction est alors $c^{\prime} E_{n} / L$. Néanmoins, cette puissance ne pourrait être extraite qu'avec un couplage parfait entre la jonction et le guide d'ondes où est placée la jonction. En réalité le couplage réalisable est, malheureusement, très mauvais. L'impédance caractéristique $Z_{J}$ de la jonction est fonction de ses dimensions :

$$
Z_{J}=\frac{c}{c^{\prime}}\left(\frac{l}{w \varepsilon}\right) Z_{0}
$$

où $w$ est sa largeur (dans le cas considéré suivant $y$ et $H_{0}$ ) et $Z_{0}$ est l'impédance de l'espace libre.

Le coefficient de transmission $T$ à la discontinuité jonction-guide d'ondes est avec une bonne approximation

$$
T=\frac{4 Z_{G} Z_{J}}{\left(Z_{G}+Z_{J}\right)^{2}}
$$

où $Z_{G} \simeq Z_{0}$ est l'impédance caractéristique du guide d'ondes.

En utilisant les ordres de grandeur caractéristiques des jonctions habituelles, $w \simeq 0,25 \mathrm{~mm}, l \simeq 10 \AA$, $\varepsilon \simeq 4$ et $c^{\prime} / c \simeq 5 \times 10^{-2}$, on trouve $T \simeq 10^{-4}$.

La puissance rayonnée, $P_{n}=c^{\prime} E_{n} T / L$, pour l'exemple considéré avec $L=1 \mathrm{~mm}$, est alors de l'ordre de $5 \times 10^{-12} \mathrm{~W}$.

En conséquence, on voit clairement que :

a) la puissance rayonnée qu'on peut espérer est très faible ;

b) le couplage joue un rôle extrêmement important et la recherche de la meilleure adaptation est indispensable ;

c) de toute façon, il faut utiliser un système de détection très sensible. 
Les expériences effectuées par Langenberg et ses collaborateurs en 1965 [2] ont permis la détection d'un. rayonnement dont la puissance est de l'ordre de $3 \times 10^{-16} \mathrm{~W}$. Il a été constaté que le rayonnement est cohérent mais pas nécessairement monochromatique. Bien que la situation soit souvent très complexe, le comportement harmonique et sous-harmonique paraît être consistant avec les résultats de calcul qu'on peut obtenir à l'aide des équations de Josephson et de Maxwell combinées.

En ce qui concerne l'utilisation d'une jonction Josephson comme source de rayonnement hyperfréquence, nous pouvons faire les remarques suivantes :

1) La limite de fréquence supérieure, $v_{\max }$ paraît être imposée par la condition $e V_{0}=\Delta(T)$ où il se produit un phénomène d'absorption interne, les paires d'électrons de Cooper étant rompues et transformées en électrons normaux. Alors $v_{\max }$ peut être estimée à l'aide de la relation $h v=2 \Delta(T)$. Les valeurs de gap d'énergie $\Delta$ qui caractérisent les divers supraconducteurs à des températures nettement inférieures à leurs températures critiques $T_{\mathrm{c}}$ conduisent alors aux valeurs $v_{\max }$ estimées de l'ordre de $350 \mathrm{GHz}$ pour l'étain, de $650 \mathrm{GHz}$ pour le plomb et de 800 à $1000 \mathrm{GHz}$ pour certains alliages, comme p. e. $\mathrm{Pb}-\mathrm{Bi}$.

La gamme d'hyperfréquences de 100 à $1000 \mathrm{GHz}$ correspond aux longueurs d'ondes millimétrique et sub-millimétrique pour lesquelles un générateur à jonction Josephson de conception simple présente un intérêt certain.

2) La riche teneur en harmoniques et en sousharmoniques du rayonnement détectable peut être avantageusement exploitée. On peut faire varier la puissance émise aux différentes fréquences en ajustant $H_{0}$, qui agit sur le couplage des différents modes de la jonction.

3) La fréquence de sortie d'un tel dispositif est facilement réglable à l'aide de la tension de polarisation. Néanmoins, pour une seule jonction, le réglage n'est pas continu, mais se limite à des bandes de fréquence étroites autour de valeurs discrètes successives.

4) La puissance hyperfréquence de sortie qu'on peut obtenir pratiquement est très faible. Par conséquent, les problèmes d'adaptation doivent être très sérieusement étudiés. On pourrait également envisager la fabrication et l'exploitation simultanées d'un grand nombre de jonctions en parallèle.

5) La pureté spectrale, grande stabilité et très faible bruit (dus à l'impédance et à la température d'utilisation très faibles), qui caractérisent les jonctions Josephson, présentent un intérêt d'utilisation tout particulier.

2. Détection de rayonnement hyperfréquence. - Si une jonction est soumise simultanément à une tension de polarisation $V_{0}=$ Cte et une irradiation hyperfréquence de pulsation $\omega=2 \pi v$, une tension $v \cos \omega t$ due à cette irradiation appliquée de l'extérieur va se superposer à la tension $V_{0}$ aux bornes de la jonction.

Alors l'expression du courant $I$ qui passe à travers'la jonction, déduite des équations (1) et (2), peut être mise sous la forme suivante :

$$
I=I_{1} \sin \left[\varphi_{0}+\frac{2 e V_{0}}{\hbar} t+\frac{2 e v}{\hbar \omega} \sin \omega t\right] .
$$

En développant cette expression en fonction de Bessel, on obtient :

$$
\begin{aligned}
I=I_{1} \sum_{n=-\infty}^{\infty}(-1)^{n} J_{n}\left(\frac{2 e v}{\hbar \omega}\right) \times & \\
& \times \sin \left(\frac{2 e V_{0}}{\hbar} t-n \omega t+\varphi_{0}\right)
\end{aligned}
$$

où $J_{n}$ représente la fonction de Bessel d'ordre $n$.

$\mathrm{Au}$ cas où la tension de polarisation $V_{0}$ est choisie telle que $2 \mathrm{eV} / \hbar=n \omega$, alors le courant Josephson a une composante continue, $I_{\text {cont }}$. Avec l'hypothèse de $v \ll V_{0}, I_{\text {cont }}$ s'écrit :

$$
I_{\mathrm{cont}} \simeq I_{1}(-1)^{n} J_{n}\left(\frac{2 e v}{\hbar \omega}\right) \sin \varphi_{0} .
$$

Cette relation signifie que dans ces conditions on peut faire varier le courant à travers la jonction entre les limites $\pm I_{1} J_{n}(2 \mathrm{ev} / \hbar \omega)$ sans que la tension de polarisation continue change de valeur.

Les expériences confirment que pour une série de tensions de polarisation continues $V_{n}=n h v / 2 e$, des marches de courant à tension constante apparaissent sur la caractéristique $I-V$ de la jonction.

Il est à remarquer que la pente $\mathrm{d} I / \mathrm{d} V$ des marches d'escalier qu'on peut obtenir lors de la génération de rayonnements hyperfréquences n'est pas infinie (la différence de tension entre les extrémités d'une marche est de l'ordre de $1 \mu \mathrm{V}$ dans les cas typiques), tandis que dans le cas d'irradiation extérieure elle est infinie, tout au moins dans les conditions idéales. Les marches légèrement inclinées trouvées dans certaines conditions expérimentales sont attribuables aux effets de bruit. Elles se manifestent surtout aux marches d'ordre élevé. Néanmoins, il a été vérifié expérimentalement que la tension $V_{n}$ du point milieu entre les deux extrémités de la $n$-ième marche est bien reliée à la fréquence d'irradiation par un multiple $(n)$ du rapport $h / 2 e$. Ce rapport est une constante physique indépendante $\mathrm{du}$ ou des matériaux supraconducteurs, de la température, du champ magnétique et de la façon dont le couplage des supraconducteurs est réalisé.

L'apparition de ces marches d'escalier sur la caractéristique $I-V$ continus peut être exploitée pour l'élaboration d'un détecteur de rayonnement hyperfréquence. La détection d'une marche définie permet la détermination de la fréquence du rayonnement grâce à la relation entre $v$ et $V$.

Du point de vue de la puissance, une détection plus sensible peut être obtenue en mesurant le changement 
du courant Josephson continu maximal dû au rayonnement appliqué, comme il est montré schématiquement sur la figure 4 pour une jonction à pointe. Le rayonnement incident sur la jonction fait décroître le courant qui le traverse et si le dispositif est alimenté par une source de courant constant, ce phénomène produit une variation de tension aux bornes de la jonction. Alors la grandeur de cette variation de tension peut être utilisée pour la mesure du rayonnement incident. Dans le cas d'un dispositif pratique, le rayonnement est échantillonné et un système de détection de phase est employé. Grimes et ses collaborateurs ont utilisé cette technique avec une jonction à pointe $\mathrm{Nb}-\mathrm{Nb}$ pour détecter un rayonnement $70 \mathrm{GHz}$ de $5 \times 10^{-13} \mathrm{~W}$ (puissance de bruit équivalente, avec une largeur de bande de $1 \mathrm{~Hz}$ ) [3].

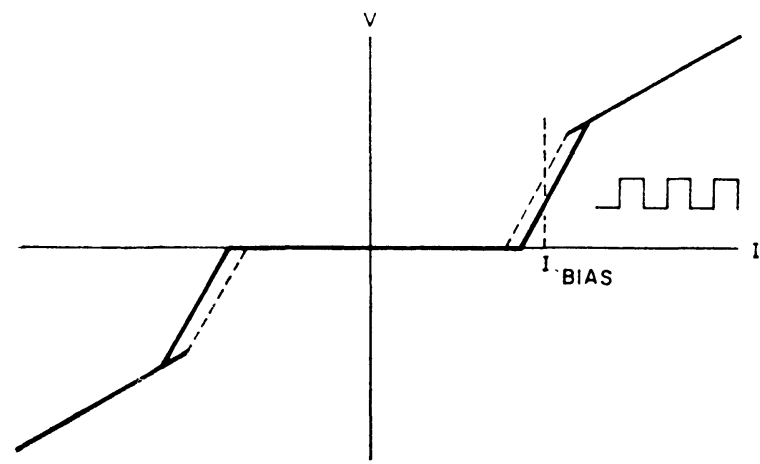

FIG. 4. - Caractéristique $V-I$ schématisée d'une jonction. Le trait épais représente l'allure en absence de rayonnement hyperfréquence tandis que le trait pointillé indique la modification sous l'influence d'un rayonnement appliqué. La jonction étant alimentée par une source de courant constant, une variation de tension aux bornes de la jonction est obtenue.

Egalement Grimes et ses collaborateurs ont montré qu'une jonction Josephson peut servir comme détecteur de rayonnement infrarouge [4] pour des rayonnements dont la longueur d'onde est de quelques centaines de $\mu \mathrm{m}$. Non seulement la sensibilité est très élevée, mais les temps de réponse très courts réalisables (de l'ordre de $10^{-8} \mathrm{~s}$ ) représentent un avantage supplémentaire. Cela permet d'échantillonner le signal incident, d'où une nette amélioration du facteur signal/ bruit.

Un terrain d'application tout particulier de ce genre de détecteurs est l'astronomie infrarouge à jonction Josephson, comme en témoignent les travaux de B. T. Ulrich de l'Université de Texas, de Shapiro à l'Université de Rochester (N. Y.) et de Mercereau à California Institute of Technology. C'est M. Ulrich lui-même qui va parler dans la suite de ce sujet très intéressant.

La relation fondamentale reliant une tension à une fréquence est exploitée pour un usage métrologique particulier qui est la surveillance des piles étalon de tension. Pour cette application on tire profit du fait que les fréquences peuvent être mesurées relativement facilement avec une très grande précision (jusqu'à $10^{-11}$ près).
La figure 5 donne la représentation schématique d'un montage de mesures approprié. Supposons que les caractéristiques intrinsèques des jonctions Josephson utilisées soient telles qu'on puisse toujours créer une marche de courant bien définie à une certaine tension, par exemple autour de $1 \mathrm{mV}$ (La fréquence de l'irradiation étant de $10 \mathrm{GHz}$ par exemple, $1 \mathrm{mV}$ correspond à la $50^{\mathrm{e}}$ marche environ). Dans ces conditions, on peut envisager la construction d'un diviseur de tension de rapport fixe, égal au rapport de la tension de la pile étalon et de la tension de polarisation prévue, c'est-à-dire dans notre exemple égal à 1000 environ.

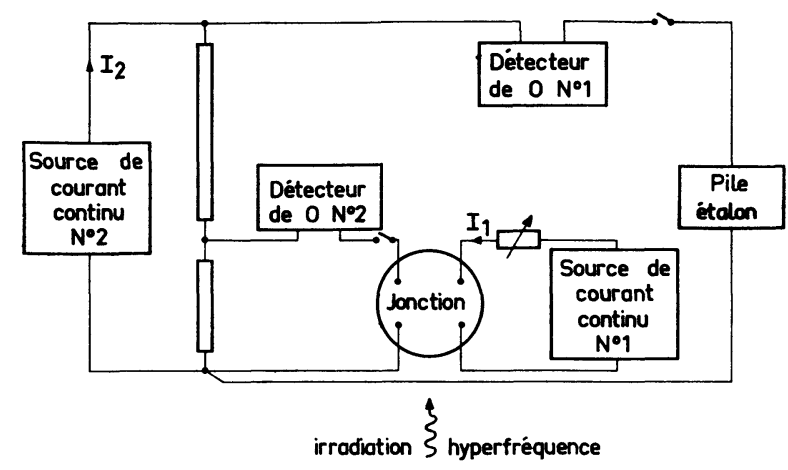

FIG. 5. - Représentation schématique d'un montage de mesures pour la surveillance des piles étalon de tension.

On procède alors de la façon suivante : à l'aide des caractéristiques $I-V$ continus, on détermine d'abord le courant $I_{1}$ nécessaire à l'obtention de la tension de polarisation envisagée et on ajuste la source de courant continu $n^{0} 1$ en conséquence. Ensuite, l'intensité du courant passant par le diviseur et provenant d'une deuxième source de courant est ajustée pour équilibrer la tension aux bornes du diviseur à l'aide du détecteur de zéro $\mathrm{n}^{0} 1$. En maintenant les valeurs de courants $I_{1}$ et $I_{2}$ on procède à l'équilibrage à l'aide du détecteur de zéro $\mathrm{n}^{0} 2$, la tension de polarisation de la jonction étant cette fois-ci précisément ajustée par modification convenable de la fréquence de l'irradiation hyperfréquence.

A l'aide de ces opérations successives et après avoir appliqué les corrections appropriées, le rapport $2 \mathrm{e} / \mathrm{h}$ peut être déduit en termes de force électromotrice de la pile étalon. Les mesures étant répétées en fonction du temps et en considérant que $2 e / h$ est une constante physique absolument invariable, tout changement dans le rapport fréquence-tension doit être attribué au changement dans le temps de la force électromotrice de la pile étalon étudiée.

Quand il s'agit de comparer les piles étalon de différents laboratoires, on peut abandonner l'ancienne pratique qui consistait à déplacer les étalons d'un laboratoire à l'autre. Avec la méthode décrite ci-dessus, les divers laboratoires peuvent effectuer les mesures indépendamment, leurs étalons de tension pouvant être comparés par l'intermédiaire de leurs étalons de fréquence. 
Les travaux relatifs à cette application sont particulièrement avancés au N. B. S. aux U. S. A. [5], [6], [7] et au N. P. L. en Angleterre [8] et ils ont été entrepris au L. C. I. E. également.

L'extension de cette utilisation est l'idée de réaliser des sources de tension Josephson. A ce propos, il est à signaler que les chercheurs du N. B. S. ont déjà réussi à produire des sources de tension stables de $10 \mathrm{mV}$ utilisant soit une seule jonction, soit plusieurs jonctions en série.

3. Mélange de rayonnements hyperfréquences. Grimes et Shapiro ont étudié [9] théoriquement et expérimentalement les conséquences de l'irradiation d'une jonction à pointe simultanément par deux rayonnements hyperfréquences.

Pour les calculs, on utilise les équations fondamentales (1) et (2) avec les hypothèses qu'aucun champ magnétique n'est appliqué à la jonction et que $\varphi$ ne varie pas dans l'espace. La présence simultanée d'une tension de polarisation continue $V_{0}$ et de deux tensions $v_{1}$ et $v_{2}$ de pulsation $\omega_{1}$ et $\omega_{2}$, respectivement, dues à deux rayonnements hyperfréquences, conduit à l'expression suivante de la tension totale $V$ aux bornes de la jonction

$$
V=V_{0}+v_{1} \cos \left(\omega_{1} t+\theta_{1}\right)+v_{2} \cos \left(\omega_{2} t+\theta_{2}\right)
$$

où les angles $\theta_{1}$ et $\theta_{2}$ sont pris par rapport à $t=0$. Avec l'hypothèse supplémentaire que $\omega_{1}$ et $\omega_{2}$ sont sensiblement différentes et avec la relation $\omega_{0}=2 \mathrm{eV} / \hbar$, on obtient alors

$$
\begin{aligned}
\frac{j}{j_{1}}=\sin \left[\omega_{0} t+\varphi_{0}+\frac{2 e v_{1}}{\hbar \omega_{1}}\right. & \sin \left(\omega_{1} t+\theta_{1}\right)+ \\
& \left.+\frac{2 e v_{2}}{\hbar \omega_{2}} \sin \left(\omega_{2} t+\theta_{2}\right)\right] .
\end{aligned}
$$

Le développement de cette expression en fonctions de Bessel conduit à

$$
\begin{aligned}
\frac{j}{j_{1}}= & \sum_{k=-\infty}^{\infty} \sum_{l=-\infty}^{\infty} J_{k}\left(\frac{2 e v_{1}}{\hbar \omega_{1}}\right) J_{l}\left(\frac{2 e v_{2}}{\hbar \omega_{2}}\right) \times \\
& \times \sin \left[\omega_{0} t+\varphi_{0}+k\left(\omega_{1} t+\theta_{1}\right)+l\left(\omega_{2} t+\theta_{2}\right)\right]
\end{aligned}
$$

où $J_{n}(X)$ est la fonction de Bessel d'ordre $n$.

D'après cette expression, on prévoit sur la caractéristique $I-V$ continus de la jonction l'apparition de marches de courant à tension constante non seulement pour $\omega_{0}=k \omega_{1}$ et $\omega_{0}=l \omega_{2}$, mais également pour $\omega_{0}=k \omega_{1} \pm m \Delta \omega \quad$ et $\quad \omega_{0}=l \omega_{2} \pm n \Delta \omega$, où $\Delta \omega=\omega_{1}-\omega_{2}$ et $k, l, m$ et $n$ sont des nombres entiers.

Les expériences effectuées ont confirmé l'existence de ce mécanisme de mélange Josephson. La figure 6, extraite de [9], montre des caractéristiques relevées expérimentalement, où on distingue nettement les marches additionnelles correspondant à $\Delta \omega$ et à ses multiples, groupées autour des marches associées

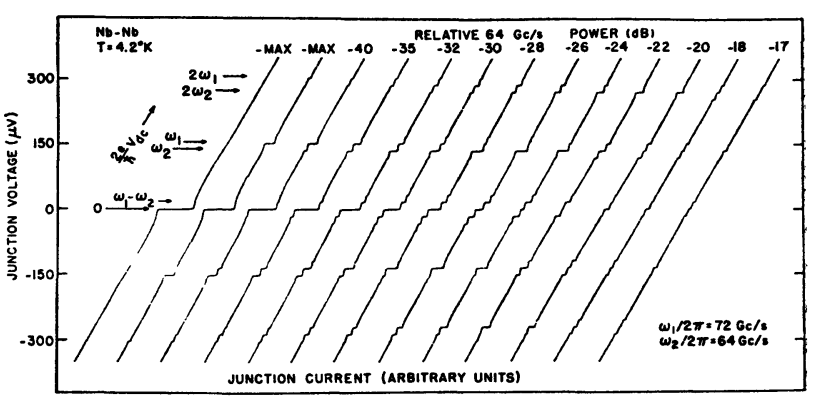

FIG. 6. - Caractéristiques $V-I$ en absence d'irradiation hyperfréquence (première courbe à gauche) et en présence de deux irradiations simultanées, l'une de $72 \mathrm{GHz}$ à puissance constante, l'autre de $64 \mathrm{GHz}$ à puissance variable.

directement à $\omega_{1}$ et $\omega_{2} \quad\left(\omega_{1} / 2 \pi=72 \mathrm{GHz}\right.$ et $\omega_{2} / 2 \pi=64 \mathrm{GHz}$ ). La figure 7, extraite également de [9], est relative aux résultats d'une expérience, où deux rayonnements d'environ $72 \mathrm{GHz}$ ont été appliqués, la différence entre les deux fréquences étant environ $1 \mathrm{MHz}$. Dans ce cas, les deux signaux appliqués, de fréquences $f_{1}$ et $f_{2}$ presque égales, produisent un battement. En ce qui concerne leurs effets sur la caractéristique $I-V$ et sur le spectre de fréquence du courant Josephson, ils sont équivalents à un signal appliqué unique, dont l'amplitude est modulée à la fréquence $f_{1}-f_{2}$. La jonction réagit sur un signal de puissance quasi statique et elle suit facilement les lentes variations de puissance, d'où l'effet de démodulation. Le signal
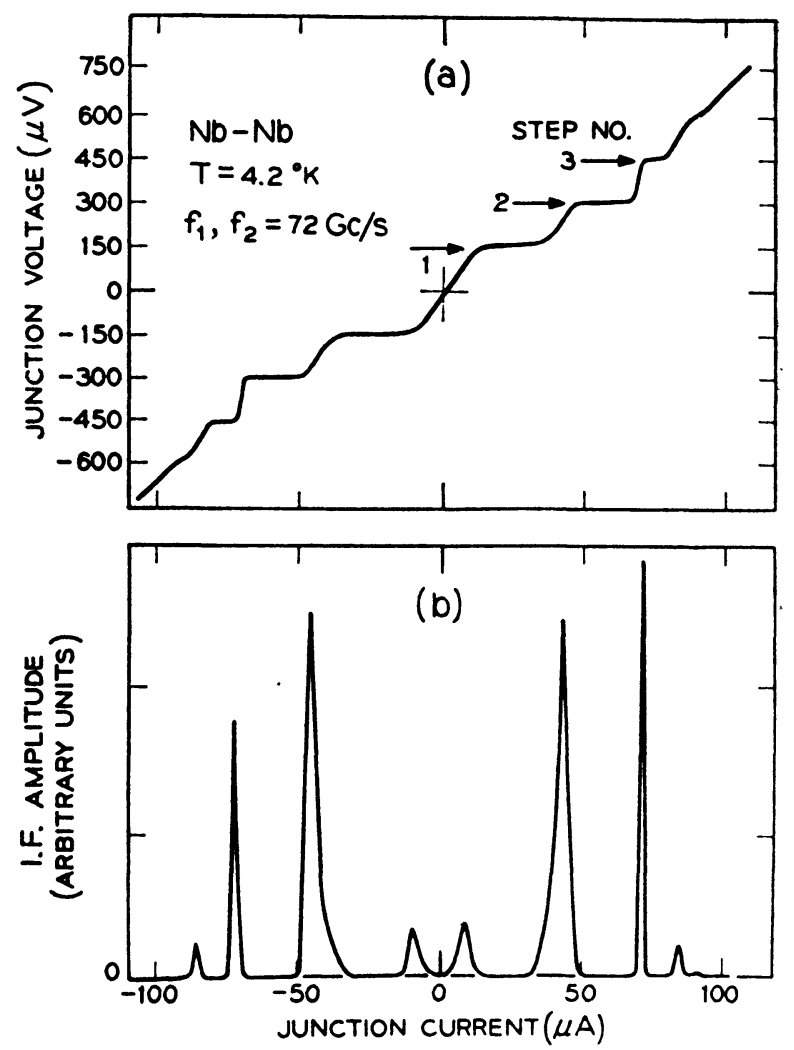

FIG. 7. - Pour une jonction irradiée simultanément par deux rayonnements d'environ $72 \mathrm{GHz}: a$ ) la tension aux bornes de la jonction et $b$ ) l'amplitude du signal de sortie à la fréquence intermédiaire de $1 \mathrm{MHz}$, en fonction du courant continu à travers. la jonction. 
de sortie à la fréquence intermédiaire $(1 \mathrm{MHz}$ pour notre exemple) est relevé directement aux bornes de la jonction, puis amplifié à la température ambiante. La partie inférieure de la figure 7 représente le signal de sortie amplifié, puis redressé, en fonction du courant continu à travers la jonction.

Dans le mélangeur Josephson, la jonction est alimentée par une source de courant constant et la variation effective de l'amplitude du signal d'entrée produit une tension de sortie variant à la même cadence. Il a été démontré que le gain de conversion est proportionnel à la résistance différentielle de la jonction au point de fonctionnement. Par conséquent, l'effet de mélange se manifeste entre les marches, tandis que sur les marches où la résistance différentielle est essentiellement zéro, on n'observe pas de signal de fréquence intermédiaire.

Une jonction Josephson peut être utilisée comme son propre oscillateur local. Si un signal d'entrée a la pulsation $\omega_{1}$ et la jonction est polarisée de façon à avoir la pulsation propre de $\omega_{0}=\omega_{1}+\Delta \omega$, alors grâce à l'effet de mélange un signal de sortie de fréquence intermédiaire (de pulsation $\Delta \omega$ ) peut être obtenu.

Diverses applications sont envisageables grâce à des propriétés remarquables des jonctions Josephson :

a) Détection très sensible. - Puissance de bruit équivalente meilleure que $10^{-14} \mathrm{~W} / \sqrt{f}$.

b) Temps de réponse très rapide. - Pour les jonctions à pointe il est inférieur à $10 \mathrm{~ns}$, par conséquent $\Delta f \leqslant 100 \mathrm{MHz}$ semble être réalisable.

c) Gamme de fréquence d'utilisation très large. - Silver et Zimmerman ont exploité une jonction comme oscillateur et en même temps comme mélangeur [10]. Une jonction étant soumise à un signal de $30 \mathrm{MHz}$ ils ont détecté une oscillation à la fréquence intermédiaire de $0,2 \mathrm{~Hz}$, correspondant à une tension de polarisation continue de $4 \times 10^{-15} \mathrm{~V}$.

A l'autre extrême, Mc Donald et ses collaborateurs ont observé [11] le mélange de deux signaux produits par laser à des fréquences peu inférieures à $900 \mathrm{GHz}$. La limite supérieure possible semble être encore plus élevée, puisqu'ils ont détecté des oscillations de jonction à $8 \mathrm{THz}(=8000 \mathrm{GHz})$ correspondant à une structure de marche d'escalier sur la caractéristique $I-V$ à des tensions de polarisation atteignant $17 \mathrm{mV}$.

M. Combet va nous mettre au courant des études en cours au C. N. E. T. sur des mélangeurs à effet Josephson.

4. Thermométrie de bruit. - Etant donné que la fréquence d'oscillation d'une jonction Josephson est déterminée par sa tension de polarisation, si cette tension est soumise à des variations dues au bruit thermique, alors ce bruit va produire des variations dans la fréquence d'oscillation. La mesure de ces variations de fréquence constitue la base d'une thermométrie absolue, élaborée au N. B. S. par Kamper et ses collaborateurs [12]. Le principe en est le suivant : un shunt de très faible valeur de résistance $\left(R=10^{-5} \Omega\right.$ environ) est connecté en parallèle à une jonction Josephson. Le shunt étant traversé par un courant $I$ de très faible intensité, la tension $V$ aux bornes de la jonction sera $V=I R+$ bruit, et les variations correspondantes dans la fréquence d'oscillation de la jonction (déterminées par la relation $f=2 \mathrm{eV} / \mathrm{h}$ ) permettent la détermination de la température absolue de $R$. Dans l'arrangement élaboré par Kamper, la jonction joue le rôle d'un préamplificateur dont la température de bruit est inférieure à celle à mesurer et présente un gain suffisant à la génération d'un signal détectable par un récepteur à la température ambiante.

Une méthode pour mesurer les fluctuations de fréquence consiste à utiliser un analyseur de spectre et à déterminer la largeur de ligne $\Delta f$ de l'oscillation, qui permet de mesurer la température absolue, grâce à la relation suivante : $\Delta f=\left(4,03 \times 10^{7}\right) R T$.

Une autre technique, qui a été retenue par Kamper, utilise un compteur de fréquence. Ce dispositif compte successivement le nombre des cycles dans un intervalle de temps, $\tau$ fixe. La variance, ou déviation quadratique moyenne $\sigma^{2}$ est alors reliée à $T$ par la formule

$$
\sigma^{2}=\left\langle(f-\bar{f})^{2}\right\rangle=6,4 \times 10^{6} \frac{R T}{\tau} .
$$

Les avantages de cette méthode sont la possibilité de : a) automatiser l'enregistrement des données et b) éliminer certaines sources de bruit perturbatrices.

Le schéma de principe utilisé pour le prototype du thermomètre réalisé au N. B. S. est représenté sur la figure 8. Des températures aussi faibles qu'au moins $75 \mathrm{mK}$ ont été déjà enregistrées avec cet appareil.

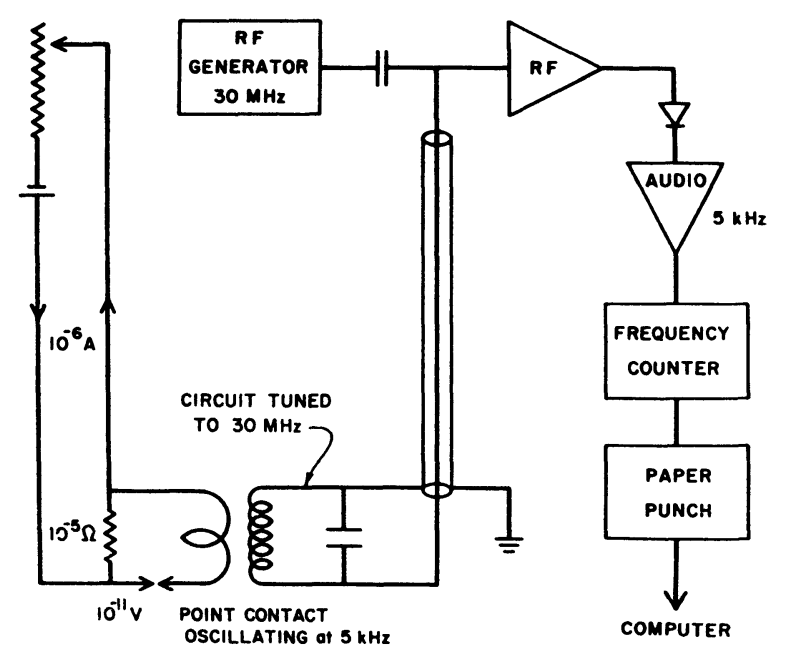

FIG. 8. - Schéma de principe du thermomètre de bruit réalisé au N.B.S.

L'intérêt particulier de ce genre de dispositif est que des mesures peuvent être effectuées avec une seule jonction dans une très large gamme de températures : de la région des $\mathrm{mK}$ jusqu'à la température critique $T_{\mathrm{c}} \mathrm{du}$ supraconducteur utilisé pour la jonction (pour le niobium $T_{\mathrm{c}} \simeq 9^{\circ} \mathrm{K}$ ). 


\section{Bibliographie}

[1] ECK (R. E.), Scalapino (D. J.) and Taylor (B. N.). Self detection of the a.c. Josephson current, Phys. Rev. Letters, 1964, 13, 15.

[2] Langenberg (D. N.), Scalapino (D. J.) and Taylor (B. N.), Josephson-type superconducting tunnel junctions as generators of microwave and submillimeter wave radiation, Proc. I. E. E. E., 1966, $54,560$.

[3] Grimes (C. C.), Richards (P. L.) and Shapiro (S.), Far infrared response of point-contact Josephson junctions, Phys. Rev. Letters, 1966, 17, 431.

[4] Grimes (C. C.), Richards (P. L.) and Shapiro (S.), Josephson effect far-infrared detector, J. Appl. Phys., 1968, 39, 3905.

[5] TAYlor (B. N.), PARKer (W. H.), LANGenberg (D. N.) and Denenstein (A.), On the use of the a.c. Josephson effect to maintain standards of electromotive force, Metrologia, 1967, 3, 89.

[6] Parker (W. H.), Langenberg (D. N.), Denenstein (A.) and TAYLOR (B. N.), Determination of $e / h$, using macroscopic quantum phase coherence in superconductors., I. Experiment., Phys. Rev., 1969, $177,639$.

[7] Finnegan (T. F.), Denenstein (A.) and Langenberg (D. N.), A. c. Josephson effect determination of $e / h$ with sub-part-per million accuracy, Phys. Rev. Letters, 1970, 24, 738.

[8] Petley (B. W.) and Morris (K.), A measurement of $2 e / h$ by the a. c. Josephson effect, Metrologia, $1970,6,46$.

[9] Grimes (C. C.) and ShapIRo (S.), Millimeter wave mixing with Josephson junctions, Phys. Rev., 1968, $169,397$.

[10] Zimmerman (J. E.) and Silver (A. H.), A high sensitivity superconducting detector, J. Appl. Phys., 1968, 39, 2679.

[11] Mc Donald (D. G.), Kose (V. E.), Evenson (K. M.), WeLls (J. S.) and CUPP (J. D.), Harmonic generation and submillimeter wave mixing with the Josephson effect, Appl. Phys. Letters, 1969, 15, 121.

[12] KAMPER (R. A.) and Zimmerman (J. E.), Noise thermometry with the Josephson effect, J. Appl. Phys., 1971, 42, 132. 